PROCEEDINGS OF THE

AMERICAN MATHEMATICAL SOCIETY

Volume 127, Number 9, Pages 2755-2758

S 0002-9939(99)04916-3

Article electronically published on April 23, 1999

\title{
A CLASS OF 3-DIMENSIONAL MANIFOLDS WITH BOUNDED FIRST EIGENVALUE ON 1-FORMS
}

\author{
GIOVANNI GENTILE
}

(Communicated by Peter Li)

\begin{abstract}
Let $(P, g)$ be the framebundle over an oriented, $C^{\infty}$ Riemannian surface $S$. Denote by $\lambda-1,1(P, g)$ the first nonzero eigenvalue of the Laplace operator acting on differential forms of degree 1 . We prove that $\lambda_{1,1}(P, g) \leq c$ for all $(P, g)$ with canonical metrics $g$ of volume 1 .
\end{abstract}

\section{INTRODUCTION}

Let $\left(M^{n}, g\right)$ be a compact, connected Riemannian manifold of $n$ dimensions. The Laplacian $\Delta_{g, p}$ acting on differential forms of degree $p$ on $M$ has discrete spectrum. Let $\lambda_{1, p}(g)$ denote the smallest positive eigenvalue of $\Delta_{g, p}$. For functions we set as usual $\lambda_{1}(g)=\lambda_{1,0}(g)$. Let $\Omega$ be the class of all metrics on an orientable closed surface $S$ with given volume. Then we have

$$
\lambda_{1}(g) \leq \frac{8 \pi(\gamma+1)}{\operatorname{Vol}(S, g)}
$$

for any Riemannian metric $g \in \Omega$. This was proved by J. Hersch [7] (for $S^{2}$ ) and by P. Yang and S.-T. Yau [14] for surfaces of higher genius.

In connection with this result, M. Berger [1] and S. Tanno [11] asked whether there exists a constant $k(M)$ such that

$$
\lambda_{1, p}(g) \leq \frac{k(M)}{\operatorname{Vol}\left(M^{n}, g\right)^{2 / n}}
$$

for any Riemannian metric $g$ on $M$.

The answer is negative in the case of functions for $n \geq 3$ (Bleecker [3], Urakawa [12], $\mathrm{Xu}$ [13], Colbois and Dodziuk [5]), and also negative in the case of differential forms of degree $2 \leq p \leq n-2$ and dimension $n \geq 4$ (Gentile and Pagliara [6]).

One can obtain positive answers if one restricts the class of manifolds $(M, \Omega)$. For $\Omega$ the class of all Kähler metrics on a complex manifold $M$, whose Kähler form represents a given cohomology class, an upper bound for $\lambda_{1}(M, g)$, i.e. functions, $g \in \Omega$ exists and is related to the algebraic-geometric properties of $M$. This was discovered by P. Li and S.-T. Yau [8] and by J.-P. Bourguignon, P. Li and S.-T. Yau [4]. Another positive answer for an $(M, \Omega)$ was given by L. Polterovich [10], when $M$ is a closed symplectic manifold and $\Omega$ a special class of Kähler metrics.

Received by the editors December 1, 1997.

1991 Mathematics Subject Classification. Primary 53C20; Secondary 58G25.

(C)1999 American Mathematical Society 
In the present paper we give a positive answer to the above problem on 1forms in a special class of 3-dimensional manifolds. They are $S^{1}$-bundles $P$ over 2 -dimensional manifolds $S$ with canonical metrics (see below). We estimate $\lambda_{1,1}(g)$ form above in terms of the fibrelength and the volume of the base-space $S$. In particular $\lambda_{1,1}(g)$ is bounded from above by a constant if we fix the volume of $P$, in contrast to the case of function, where D. Bleecker [3] showed, that $\lambda_{1}(g)$ cannot be bound by a constant in this class of manifolds.

\section{THE CLASS OF MANIFOLDS}

Let $P$ be the framebundle over an oriented closed Riemannian surface $(S, h)$. This is a principal $S^{1}$-bundle. An $S^{1}$-invariant metric on the fibre and the metric $h$ of the base space $S$ induce a Riemannian metric $g$ on $P$ such that $\pi:(P, g) \rightarrow(S, h)$ is a Riemannian submersion with totally geodesic fibres and horizontal distribution associated with the Levi-Civita connection (A. Besse [2]).

Let $\xi$ be the Killing vectorfield along the $S^{1}$-action and $\eta$ its dual. Let $x=$ $\left(X_{1}, X_{2}\right)$ be a frame at $u=\pi(x)$. Each $x \in P$ gives rise to a linear isomorphism $x: \mathbb{R}^{2} \rightarrow T_{u}, u=\pi(x), x\left(E_{i}\right)=X_{i}$ where $\left(E_{1}, E_{2}\right)$ is the standard basis of $\mathbb{R}^{2}$. Let $V \in \mathbb{R}^{2}, x \in P, B(V)_{x}:=$ unique horizontal vector at $x$ which projects on $x(V) \in T_{u} . B(V)$ is called a basic vectorfield (K. Nomizu [9]).

Lemma 1. i) $V \neq 0 \rightarrow B(V) \neq 0$.

ii) $\left(\xi, B\left(E_{1}\right), B\left(E_{2}\right)\right)$ gives rise to a complete parallelisation of $P$.

iii) $[\xi, B(V)]=B(\xi \cdot V)$, where the dot denotes standard representation of $\xi \in L S^{1}$ on $\mathbb{R}^{2}$ :

$$
\xi \mapsto\left(\begin{array}{cc}
0 & -1 \\
1 & 0
\end{array}\right) .
$$

The proof of this and the following lemma can be found in K. Nomizu [9, page $50-52]$. Let $\left(\xi, B_{1}, B_{2}\right)$ be an ONS for $g, B_{i}=B\left(E_{i}\right)$ and $\Omega$ the curvature form of the fibration.

Since the torsion vanishes we have:

Lemma 2. i) $\left[B_{1}, B_{2}\right]=-\Omega\left(B_{1}, B_{2}\right)$.

ii) $\left[\xi, B_{1}\right]=B\left(\xi \cdot E_{1}\right)=B\left(E_{2}\right)=B_{2}$.

iii) $\left[\xi, B_{2}\right]=B\left(\xi \cdot E_{2}\right)=B\left(-E_{1}\right)=-B_{1}$.

\section{REsults}

Theorem 1. Let $(P, g)$ be as above. Then

$$
\lambda_{1,1}(g) \leq \min \left(\frac{1}{L^{2}}, \frac{8 \pi(1+\gamma)}{\operatorname{Vol}(S, h)}\right)
$$

where $2 \pi L$ is the length of the fibres. In particular if we fix $\operatorname{Vol}(P, g)=1$, then

$$
\lambda_{1,1}(g) \leq\left[16 \pi^{2}(1+\gamma)\right]^{2 / 3} .
$$

Remarks. If $\operatorname{Vol}(P, g)=1, \lambda_{1}(g)=\lambda_{1,0}(g)$ is not bounded as long as the curvature of $(S, h)$ is nowhere 0 (D. Bleecker [3]).

Proof. The metrics on $P$ are determined up to the length of the fibres from the metric on $S$ and the condition imposed on $P$ (A. Besse [2]). We want to examine the dependence of the eigenvalue on the length of the fibres. By rescaling the metric on $P$ along the fibres while keeping it constant on the orthogonal complement 
(canonical variation), we obtain a 1-parameter family of metrics $g_{L}$ on $P$ for which $\pi:\left(P, g_{L}\right) \rightarrow(S, h)$ is a Riemannian submersion and the length of the fibres is $2 \pi L$.

If $\left(\xi, B_{1}, B_{2}\right)$ is an ONS for $g$ and $\bar{\xi}=\frac{1}{L} \xi$, then $\left(\bar{\xi}, B_{1}, B_{2}\right)$ is an ONS for $g_{L}$. Let $\left(\bar{\eta}, \beta_{1}, \beta_{2}\right)$ be the dual base.

Lemma 3. $\beta_{1}$ is an eigen 1-form of the Laplace operator on $M$

$$
\begin{aligned}
d \beta_{1} & =-\beta_{1}\left(\left[\bar{\xi}, \beta_{1}\right]\right) \bar{\eta} \wedge \beta_{1}-\beta_{1}\left(\left[\bar{\xi}, \beta_{2}\right]\right) \bar{\eta} \wedge \beta_{2} \\
& =-\frac{1}{L} \beta_{1}\left(\left[\xi, \beta_{2}\right]\right) \bar{\eta} \wedge \beta_{2} \\
& =\frac{1}{L} \bar{\eta} \wedge \beta_{2}
\end{aligned}
$$

where we have used Lemma 2.

$$
\begin{aligned}
\delta \beta_{1} & =* d * \beta_{1} \\
& =-* d\left(\bar{\eta} \wedge \beta_{2}\right) \\
& =-*\left(d \bar{\eta} \wedge \beta_{2}-\bar{\eta} \wedge d \beta_{2}\right) \\
& =0
\end{aligned}
$$

again from Lemma 2. Therefore we get

$$
\begin{aligned}
\Delta \beta_{1} & =(\delta d+d \delta) \beta_{1} \\
& =* d * d \beta_{1} \\
& =* d *\left(\frac{1}{L} \bar{\eta} \wedge \beta_{2}\right) \\
& =-\frac{1}{L} * d \beta_{1} \\
& =\frac{1}{L^{2}} \beta_{1} .
\end{aligned}
$$

In the same way one finds that $\beta_{2}$ is also an eigen 1 -form with eigenvalue $1 / L^{2}$.

On the other hand, if $\Psi$ is a nontrivial eigenfunction of $(S, h)$, then, using the result of Yang and Yau, we have (1) and $\Delta \circ d=d \circ \Delta$

$$
\Delta^{S}(d \Psi) \leq \frac{8 \pi(1+\gamma)}{\operatorname{Vol}(S, h)} d \Psi
$$

We lift the 1 -form $d \Psi$ of $S$ to $P$. This gives (since $\operatorname{dim} S=2$ )

$$
\Delta^{P}\left(\pi^{*}(d \Psi)\right)=\pi^{*}\left(\Delta^{S}(d \Psi)\right) \leq \frac{8 \pi(1+\gamma)}{\operatorname{Vol}(S, h)} \pi^{*}(d \Psi) .
$$

Putting it together, we get

$$
\lambda_{1,1}(g) \leq \min \left(\frac{1}{L^{2}}, \frac{8 \pi(1+\gamma)}{\operatorname{Vol}(S, h)}\right)
$$

If we fix $\operatorname{Vol}(P, g)=1$, then $\operatorname{Vol}(S, h)=\frac{1}{2 \pi L}$ and the second assertion follows from

$$
\min \left(\frac{1}{L^{2}}, 16 \pi^{2}(1+\gamma) L\right) \leq\left[16 \pi^{2}(1+\gamma)\right]^{2 / 3} \quad \forall L>0
$$


Remarks. If one takes as class $(P, \Omega)$ the framebundles over manifolds $M$ of arbitrary dimension $n$, with certain canonical metrics on $S O(N)$, one can still find an analogue of $\beta_{1}$, but there is no result which corresponds to the equality of HerschYang-Yau (1). Also one does not know whether or not the first eigenvalue on functions is bounded in that class.

\section{REFERENCES}

[1] M. Berger, Sur les premières valeurs propres des variétés riemanniennes, Compositio Math. 26 (1973), 129-149. MR 47:5461

[2] A. Besse, Einstein manifolds, Springer 1987. MR 88f:53087

[3] D. Bleecker, The spectrum of a Riemannian manifold with a unit Killing vector field, Transactions Amer. Math. Soc. 275 (1983), 409-416. MR 84c:53037

[4] J.-P. Bourguignon, P. Li and S.-T. Yau, Upper bound for the first eigenvalue of algebraic submanifolds, Comm. Math. Helv. 69 (1994), 199-207. MR 95j:58168

[5] B. Colbois and J. Dodziuk, Riemannian metrics with large $\lambda_{1}$, Proc. Amer. Math. Soc. 122 (1994), 905-906. MR 95a:58130

[6] G. Gentile and V. Pagliara, Riemannian metrics with large first eigenvalue on forms of degree p, Proc. Amer. Math. Soc. 123 (1995), 3855-3858. MR 96b:58115

[7] J. Hersch, Quatre propriétés isopérimetriques des membranes sphériques homogénes, C. R. Acad. Sci. Paris Sér. A 270 (1970), 139-144.

[8] P. Li and S.-T. Yau, A new conformal invariant and its applications to the Willmore conjecture and the first eigenvalue of compact surfaces, Invent. Math. 69 (1982), 269-291. MR 84f:53049

[9] K. Nomizu, Lie groups and differential geometry, The Math. Soc. Japan 1956. MR 18:821d

[10] L. Polterovich, Symplectic aspects of the first eigenvalue, to appear.

[11] S. Tanno, Geometric expressions of eigen 1-forms of the Laplacian on spheres, Spec. Riem. manifolds, Kaigai Publ. Kyoto (1983), 115-128.

[12] H. Urakawa, On the least positive eigenvalue of the Laplacian for compact group manifolds, J. Math. Soc. Japan 31 (1979), 209-226. MR 80e:58046

[13] Y. Xu, Diverging Eigenvalues and Collapsing Riemannian Metrics, Institute for Advanced Study, October 1992.

[14] P. Yang and S.-T. Yau, Eigenvalues of the Laplacian of compact Riemann surfaces and minimal submanifolds, Ann. Scuola Norm. Sup. Pisa 7 (1980), 55-63. MR 81m:58084

Department of Mathematics, ETH-Zentrum, HG G34, CH 8092 Zurich, Switzerland 